\title{
In Silico and in Vitro Approach for the Understanding of the Xanthine Oxidase Inhibitory Activity of Uruguayan Tannat Grape Pomace and Propolis Poliphenols
}

\author{
Elena Alvareda1,2, Federico Iribarne' ${ }^{1}$, Victoria Espinosa ${ }^{3}$, Pablo Miranda ${ }^{1}$, Daniela Santi4,5, \\ Sara Aguilera ${ }^{6}$, S. Bustos ${ }^{4,5}$, Margot Paulino Zunini ${ }^{*}$ \\ ${ }^{1}$ Centro de Bioinformática Estructural, DETEMA, Facultad de Química, Universidad de la República, Montevideo, Uruguay \\ ${ }^{2}$ Centro Universitario Región Noroeste, Sede Salto, Universidad de la República, Salto, Uruguay \\ ${ }^{3}$ Centro de Investigaciones Biomédicas y Aplicadas, Escuela de Medicina, Facultad de Ciencias Médicas, Universidad de Santiago \\ de Chile, Santiago, Chile \\ ${ }^{4}$ Departamento de Ciencias Farmacéuticas, Facultad de Ciencias Químicas, Universidad Nacional de Córdoba, Ciudad \\ Universitaria, Córdoba, Argentina \\ ${ }^{5}$ Instituto Multidisciplinario de Biología Vegetal (IMBIV-CONICET), Ciudad Universitaria, Córdoba, Argentina \\ ${ }^{6}$ Faculty of Sciences, Universidad Católica del Norte, Antofagasta, Chile \\ Email: *margot@fq.edu.uy, ${ }^{\star}$ margot.pa ulino@gm ail.com
}

How to cite this paper: Alvareda, E., Iribarne, F., Espinosa, V., Miranda, P., Santi, D., Aguilera, S., Bustos, S. and Zunini, M.P. (2019) In Silico and in Vitro Approach for the Understanding of the Xanthine Oxidase Inhibitory Activity of Uruguayan Tannat Grape Pomace and Propolis Poliphenols. Journal of Biophysical Chemistry, 10, 1-14. https://doi.org/10.4236/jbpc.2019.101001

Received: November 27, 2018

Accepted: February 25, 2019

Published: February 28, 2019

Copyright $\odot 2019$ by author(s) and Scientific Research Publishing Inc. This work is licensed under the Creative Commons Attribution International License (CC BY 4.0).

http://creativecommons.org/licenses/by/4.0/

\section{(c) (i) Open Access}

\begin{abstract}
The use of food additives with xanthine oxidase (XO) inhibitory activity offers an alternative approach to hyperuricemic and gout disease treatment, and provides an example of antioxidant nutraceutics. The in vitro and in silico XO inhibitory activity of polyphenols from Uruguayan Tannat grape pomaces and propolis extracts was evaluated as well as the scavenging capacity of said compounds. When comparing propolis and grape pomace samples, the in vitro studies demonstrated that polyphenols extracted from propolis are more active as free radical scavengers than those from Tannat grape pomace. Both natural products effectively inhibited XO but the capacity of phenols present in GP is higher than the one present in $\mathrm{P}$. The high content of anthocyanins in GP, absent in P, could account for this observation. In silico assays allowed us to determine relevant ligand-receptor interactions between polyphenols, from a database built with previously reported polyphenols from both natural products, and the active site of XO. The in silico results showed that compound (E)-isoprenylcaffeate from propolis was the best potential XO inhibitor displaying hydrophobic aromatic interaction between the conjugated ring of the caffeate moiety and polar interactions between hydroxyl groups from caffeate with the active site polar residues. Among grape po-
\end{abstract}


maces, the Cyanidin-3-O-(6-(E)-p-coumaroyl)-glucoside was the best XO inhibitor; its moiety oxychromenyl being relevant to the docking stabilization. All these results lead us to propose Uruguayan propolis and Tannat grape pomace extracts as food additives as well as phytopharmaceuticals to decrease the uric acid levels in gout disease and to act against oxidative stress.

\section{Keywords}

Propolis, Tannat Grape Pomace, Xanthine Oxidase Inhibition, Polyphenols, Functional Foods

\section{Introduction}

Xanthine oxidase (XO) participates in purine catabolism, generating uric acid as a final product and the release of reactive oxygen species (ROS) such as $\mathrm{O}_{2}^{--}$ and $\mathrm{H}_{2} \mathrm{O}_{2}$. The exacerbated synthesis of uric acid causes the development of gout disease [1] [2]. At the same time, ROS are responsible for triggering a lot of disorders such as atherosclerosis, ageing, cancer, ulcer and inflammation [3] [4] [5] [6] [7]. Current gout treatments are palliative at the level of pain and chronic inflammation, and although treatments involving xanthine oxidase inhibitors are available, serious adverse effects have been reported [8]. In this context, natural products represent an important alternative. In particular, numerous polyphenols have been reported as relevant XO inhibitors [1] [2] [9] [10] [11] [12]. In addition, recent in vitro and in silico reports on $\mathrm{XO}$ inhibition by quercetin (a natural flavonoid) gave consistent and promising information to consider this natural flavonoid as a relevant compound for preventing gout and oxidative damage [13]. The antioxidant action mechanism of quercetin and its role on the inhibition of XO catalysis was also proposed. In this sense, the binding site for quercetin was mapped near the molybdopterin cofactor where the oxidation of xanthine (natural substrate) takes place. Thereafter, the reduction of the substrate oxygen is mediated by the isoalloxazine ring of the flavin adenine dinucleotide $(\mathrm{FAD})$ center, transferring electrons and generating ROS like superoxide radical anion $\left(\mathrm{O}_{2}^{\cdot-}\right)$ or hydrogen peroxide $\left(\mathrm{H}_{2} \mathrm{O}_{2}\right)$ [13] [14].

Propolis $(\mathrm{P})$ is a natural resinous product elaborated by honeybees (Apis mellifera) that displays a lot of biological activities such as antibacterial, antiviral, antifungal, anti-inflammatory, anticancer, and antioxidant [15] [16].

Grape pomaces (GP) are a by-product of wine industry and consist mainly of peels (skins), seeds and stems. Antioxidant, antimicrobial, antimutagenic, anticarcinogenic and antilipogenic activities have been reported for GP [17] [18].

Both P and GP, have been described as important sources of bioactive compounds, most of them polyphenols [17] [19] [20] [21] [22]. In this context, Yoshizumi et al. [16] suggested that a continuous intake of Chinese propolis may be effective for the prevention and the treatment of gout and hyperuricemia. 
Furthermore, ethanolic extracts from grape pomace increase the antioxidant properties of wines [18].

In this work, we studied the total phenolic contents, the scavenging activity and, for the first time, the in vitro XO inhibitory activity of polyphenolic rich extracts from Uruguayan propolis and Tannat grape pomace. In addition, in silico assays were performed in an attempt to account for the molecular basis of the interaction between the enzyme and polyphenols-like compounds, present in the extracts according to previous reports [23] [24].

\section{Materials and Methods}

\subsection{General}

Folin-Ciocalteu' phenol reagent, 1,1-diphenyl-2-picryl-hydrazyl (DPPH'), gallic acid, ascorbic acid and sodium carbonate $\left(\mathrm{Na}_{2} \mathrm{CO}_{3}\right)$ were obtained from Sigma (Sigma-Aldrich ${ }^{\oplus} \mathrm{GmbH}$, Sternheim, Germany). Methanol was obtained from Merck ${ }^{\oplus}$. All other chemicals used were of analytical grade. For the XO inhibition, a Sigma-Aldrich Xanthine Oxidase Activity Assay Kit was used (cat. MAK078, Sigma-Aldrich ${ }^{\oplus}$, St. Louis, MO). Measures were obtained from Elisa Thermo ${ }^{\circ}$ Scientific Labsystems Multiskan ${ }^{\mathrm{TM}}$ RC.

\subsection{Samples and Extracts}

$\mathrm{P}$ and GP sampling and the respective extraction procedures were developed by our group and previously published [23] [24]. Briefly, propolis and Tannat grape pomace samples were collected in different zones form the south region of Uruguay.

\subsubsection{Propolis Extracts}

Samples of $1 \mathrm{~g}$ weight were separately extracted. Waxes were removed by Soxhlet in $200 \mathrm{~mL}$ hexane during one hour and 12 refluxes. After evaporating the hexane and drying, they were grinded and phenols were extracted with ethanol-water $(80-20 \mathrm{v} / \mathrm{v})$ during one hour at a $75^{\circ} \mathrm{C}$. Finally, $25 \mathrm{~mL}$ of extract were filtered and diluted to $50 \mathrm{~mL}$ with the same extraction solvent. The liquid extracts were stocked in dark at $4^{\circ} \mathrm{C}$ until their analysis.

\subsubsection{Grape Pomace Extracts}

Frozen grape pomace samples were submitted during 24 hours to drying in vacuum at a temperature of $60^{\circ} \mathrm{C}$. The dry grape pomace samples were grinded and stocked in darkness. Phenols were extracted by reflux in a mixture of ethanol-water $(80-20 \mathrm{v} / \mathrm{v})$ during 2 hours at $50^{\circ} \mathrm{C}$. After filtering, the extracts were conserved at $4^{\circ} \mathrm{C}$ in darkness until their use.

\subsection{Total Phenolic Content (Folin-Ciocalteau)}

Total phenolic content was determined by means of Folin-Ciocalteau reagent using the method described by Singleton et al. [25]. The results are reported as mean values of GAE (gallic acid equivalents) in $\mu \mathrm{M}$, using the calibration curve 
prepared with gallic acid standard solutions.

\subsection{DPPH Radical Scavenging Activity}

Free radical scavenging activities of extracts were analyzed, based on the principle of scavenging the DPPH (1,1-diphenyl-2-picrylhydrazyl) radical. The absorbance was measured at $517 \mathrm{~nm}$; the procedure was the same described in Shimada et al. [26]. Acid ascorbic was used as a positive control and the results are reported as mean values of grams of ascorbic acid equivalents per $100 \mathrm{~g}$ dry extract.

\subsection{In Vitro XO Inhibition Assays}

The Xanthine Oxidase activity assay kit was used according to the manufacturer instructions. The measure of $\mathrm{XO}$ activity was performed by an enzyme assay, leading to a colorimetric $(570 \mathrm{~nm})$ blue product, proportional to the generated hydrogen peroxide (cat. MAK078, Sigma-Aldrich ${ }^{\oplus}$, St. Louis, MO). Assays were performed in $15 \mathrm{~min}$ according to supplier instruction using $5 \mu \mathrm{L}$ of samples in triplicate.

\subsection{Xanthine Oxidase in Silico Assays}

\subsubsection{In Silico General Tools and Modeling}

All in silico calculations and procedures were performed with the Molecular Operating Environment $\mathrm{MOE}^{\mathrm{TM}}, 2015.10$ [27]. To build the database, 123 phenolic structures from bibliographic sources [20] [24] [28] [29] [30] [31] were modeled and refined by energy minimization, using the MMF94x force field [32]. The resulting phenolic database was previously reported [24].

The X-ray structure of mammalian xanthine oxidase complexed with quercetin (flavonoid inhibitor) at $2.0 \AA$ resolution was obtained from the RCSB Protein Data Bank (PDBid: 3NVY) [14].

\subsubsection{Xanthine Oxidase Docking Procedure}

The aforementioned crystallographic structure of the XO-quercetin complex was used as starting point for the docking procedure. As expected, one of the detected sites was the active site which accommodates quercetin, the same site identified by Hille et al. [33] next to the molybdopterin cofactor. The benzopyran moiety of quercetin is oriented between Phe914 and Phe1009 residues and the B ring is next to the molybdopterin cofactor named MTE1327-MOS1228, at the active site [14] [33].

For the docking calculations, a $4.5 \AA$ sphere centered on quercetin was considered. The docked quercetin pose overlapped nicely the ligand crystallographic pose, with a $2.8 \AA$ root mean square deviation (RMSD) when Alpha Triangle (placement), Affinity $\Delta \mathrm{G}$ (scoring) and final force field (MMFF94x) refinement were used. The docking scores were calculated with the Affinity $\Delta \mathrm{G}$ function. This scheme had been developed previously by us [12] [23] [24]. The highest ranked poses were evaluated using the score and the relative position with re- 
spect to the native ligand [14]. The graphical representations of the calculated binding poses were performed by Surface Maps, and Ligand Interaction $\mathrm{MOE}^{\mathrm{TM}}$ tools [34].

\section{Results and Discussion}

The results for the different samples of Uruguayan propolis and tannat gape pomaces are summarized in Table 1. All samples were collected from the south of Uruguay near Montevideo and then analyzed, to determine the amount of phenolic compounds. Phenolic contents were obtained by Folin-Ciocalteu and they are expressed as GAE (gallic acid equivalents) in $\mu \mathrm{M}$. DPPH. Radical scavenging activities are expressed as AEAC (ascorbic acid equivalent antioxidant capacity) in $\mu \mathrm{M}$. Table 1 also presents the in vitro XO inhibitory activity (\%) of all samples.

\subsection{Total Phenolic Content (Folin-Ciocalteau)}

Both types (e.g. propolis and grape pomaces) of hydroalcoholic extracts showed different amount of polyphenols with the same extraction mixture. Phenolic contents of propolis extracts are higher (mean $=10.9 \pm 0.3 \mathrm{GAE} \mu \mathrm{M})$, than those of grape pomace (mean $=5.5 \pm 0.9 \mathrm{GAE} \mu \mathrm{M})$, evidencing that propolis extracts present more polyphenolic content (Table 1). The GP 2010 Tannat sample (extracted in 2013) presented fewer phenols than the Tannat sample extracted in the same year that was collected (GP 2013), suggesting that the amount of polyphenols from grape pomace samples probably decreases with aging.

\subsection{DPPH Radical Scavenging Activity and Xantine Oxidase in Vitro Assays}

The evaluation of the antioxidant potential from the extracts was performed by the DPPH radical scavenging method. The results depicted in Table 1 show that the extracted polyphenols from propolis are far more active as free radical scavengers than those from grape pomace extracts. However, $\mathrm{XO}$ inhibition of $\mathrm{P}$ extracts is only slightly higher than that of GP ones (mean values of $44 \%$ and $36 \%$, respectively). Considering that the total amount of phenols in GP is half the one measured in $\mathrm{P}$, it may be suggested that the specific potency of phenols present in GP is higher than the capacity of those phenols present in P. In particular, the high content of anthocyanins in GP could account for this observation.

This hypothesis is further substantiated by previous studies that found that anthocyanins were effective XO inhibitors [18] [35] [36] [37]. At the same time, other works reported that anthoc yanins are present in GP extracts [17] [19] [23] [24]. But absent in P extracts [23] [24] [38].

Previously, it was also reported that, while the concentration of polyphenols remains constant after wine maturation, the anthocyanins concentration is diminished [39]. These finding may be also correlated with our observations in GP 
Table 1. Phenolic content ( $\mu \mathrm{M}$ GAE), radical scavenger activity ( $\mu \mathrm{M} \mathrm{DPPH})$ and XO in vitro inhibitory activity (\%) of different samples (obtained in the years indicated) of Uruguayan propolis (P) and Tannat grape pomaces (GP).

\begin{tabular}{cccc}
\hline Sample code & $\begin{array}{c}\text { GAEa } \\
\mu \mathrm{M}+\mathrm{SD}\end{array}$ & $\begin{array}{c}\text { DPPHb } \\
\mu \mathrm{M}+\mathrm{SD}\end{array}$ & $\begin{array}{c}\text { XO inhibition activity } \\
(\%)+\mathrm{SD}\end{array}$ \\
\hline P1 2008 & $10.4+0.2$ & --- & $68.8+0.9$ \\
P2 2008 & $12.3+0.1$ & $37.0+0.0$ & $41.7+0.0$ \\
P3 2008 & $14.3+0.1$ & $37.3+0.2$ & $68.1+0.6$ \\
P4 2009 & $11.0+0.1$ & $36.9+0.5$ & $39.6+0.9$ \\
P5 2009 & $13.2+0.1$ & $37.8+0.1$ & $20.8+0.4$ \\
GP 2010 Tannat & $3.3+0.5$ & $1.9+1.4$ & $15.3+0.5$ \\
(extracted in 2013) & $7.7+1.2$ & $10.3+1.4$ & $56.3+0.9$ \\
GP 2013 Tannat & $4.0+0.2$ & $16.2+1.0$ & $25.0+0.4$ \\
P6 2013 & &
\end{tabular}

${ }^{\mathrm{a}}$ Gallic acid equivalents, ${ }^{\mathrm{b}}$ Ascorbic acid equivalent antioxidant capacity (AEAC). Propolis samples were labeled with a consecutive number and with their collected and extracted year. Year of collection and extraction was the same for all samples except for sample GP 2010 which was collected in 2010 but extracted in 2013. All samples were collected from the South of Uruguay.

extracts: the decrease in $\mathrm{XO}$ inhibitory activity through time could be due to the decrease in anthocyanins concentration. However, more thorough studies are necessary to prove this hypothesis correct.

The in vitro results suggest that the extracted compounds are responsible for the XO inhibitory activity and, in turn, would be able to cause the decline in the amount of free radicals and the uric acid deposits. Indeed, the events mediated by polyphenols might cause a decrease in the evolution of gout disease, as well as the tissue damage reduction usually observed in other pathologies associated with an increase in ROS generation such as ischemia-reperfusion among others [40].

\subsection{Xanthine Oxidase in Silico Data Analysis}

Table S1 (Supplementary Material) lists the 60 best docked scores from the 123 phenolic compounds database previously found in propolis [15] [28] [29] and grapes [20]. This very same database was previously reported by us [24]. 52 of the 60 compounds belong to GP extracts. This is consistent with the in vitro results observed for GP and P extracts from the same year, which show that the former presented the best inhibition.

\subsection{Docking Calculations}

The best docked compound, the hydroxycinnamic acid derivative, ester (E)isoprenylcaffeate $\left(\right.$ score $=-5.7884 \mathrm{kcal} \cdot \mathrm{mol}^{-1}$ ) belonged to propolis extracts. When a ligand interaction plot was performed for this compound, an interesting $\pi-\pi$ stacking interaction was observed between the caffeate moiety and Phe914 at a distance of $3.75 \AA$ and next to cofactor molybdopterin (molybdenum center), 
see Figure 1. This interaction stabilizes the structure; a similar stacking was described by Wróblewski et al. [41] and by Cao et al. [14]. The isoprenyl portion appears exposed to the solvent (Figure 1).

In the Tannat GP extracts, the best docked compound was the anthocyanin named cyanidin-3-O-(6-(E)-p-coumaroyl)-glucoside. In Figure 2, the most important interactions with the XO active site are depicted. The same $\pi$ - $\pi$ stacking interaction found for the binding of ester (E)-isoprenylcaffeate was also verified here but now between the coumaroyl moiety and Phe914 at a distance of $3.70 \AA$ (also next to cofactor molybdopterin (molybdenum center). A hydrogen bonding with Thr1010 and an ionic interaction with Glu879 were also observed.

The results for both compounds in Figure 1 and Figure 2 suggest that the acceptor hydrogen bond interactions with the active site residue side chains, plus the hydrophobic factor embodied by $\pi-\pi$ interactions between Phe914 and phenolic rings, play an important role in $\mathrm{XO}$ inhibition mechanism, as was previously suggested by Cao et al. [14]. Also, a hydrophilic environment seems to be preferred for the inhibitors, next to the molybdenum center near Arg880, Ser876, Glu802 or Glu1261.

The comparison between the in silico results and the in vitro experiments for $\mathrm{XO}$ inhibition would endorse our previous hypothesis in relation to the importance of anthocyanins. In effect, even allowing for the fact that the score differences are rather small across all compounds in Table S1, anthocyanins represent over $60 \%$ of the 60 best docked compounds in XO, and half of the 10 best compounds.

\section{Conclusions}

XO inhibition and scavenging of free radicals are potentially a powerful strategy for hyperuricemia and the fight against oxidative stress.

In this work, we were able to prove that the Uruguayan P and Tannat GP extracts can act as free radical scavengers, with samples of propolis being significantly more active than those of grape pomaces. In addition, we have shown that $\mathrm{P}$ and GP samples are capable, to different extents, of inhibiting XO. However, XO inhibition by P extracts is only slightly higher than GP. Considering that the total amount of phenols in GP is half of that measured in P, we attribute these results to the anthocyanin-like compounds, only present in GP extracts. In other words, we believe that the superior inhibitor ability of GP extracts is mainly due to the high concentration of anthocyanins present in the early stages of the grape pomaces. Further experimental assays that measure the exact concentration of anthocyanins are necessary to confirm this hypothesis.

The molecular docking results were used to determine the way in which the phenolic compounds would interact with XO's active site. In effect, considering the results yielded by the best docked compounds, it can be concluded that, at the molecular level, three different types of interactions are established with the enzyme: a) hydrophobic aromatic interactions between the conjugated ring of 

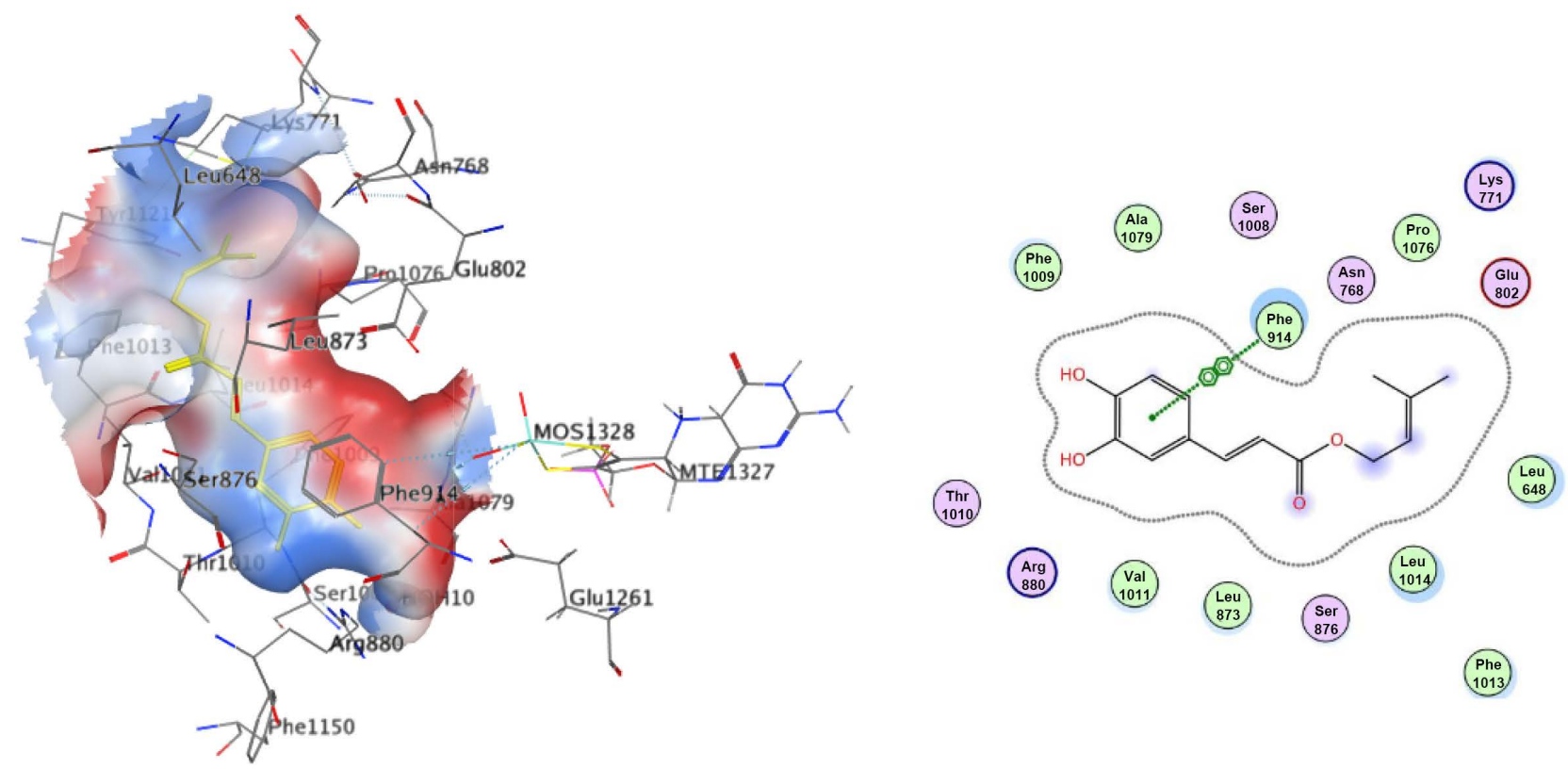

Figure 1. 2D Electrostatic Surface Map and Ligand Interaction of the best docked ligand from all samples, ester (E)-isoprenylcaffeate $\left(\right.$ score $=-5.7884 \mathrm{kcal} \cdot \mathrm{mol}^{-1}$ ) displayed in yellow.
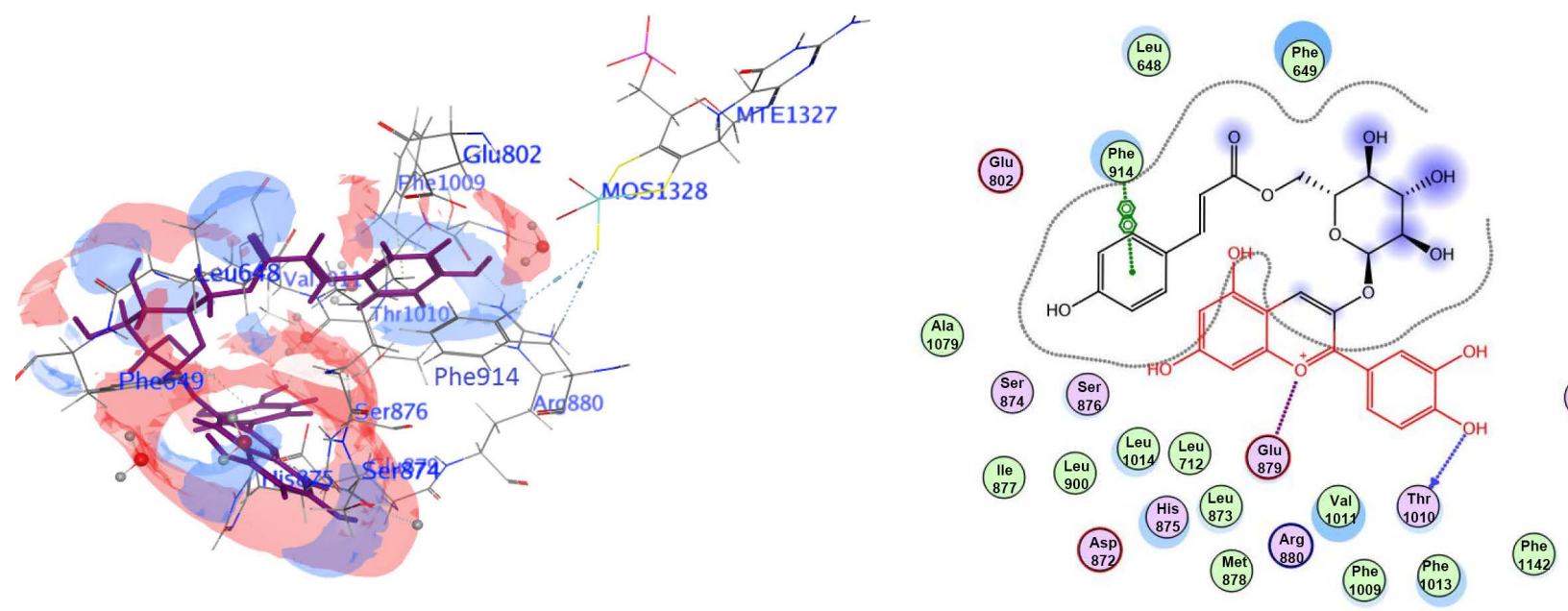

Figure 2. 2D Electrostatic Surface Map and Ligand Interaction of the best docked compound from GP extracts, Cyanidin-3-O-(6-(E)-p-coumaroyl)-glucoside, displayed in violet.

the caffeate moiety and the conjugated coumaroyl ring, with the active site phenylalanine residues; b) polar interactions between hydroxyl groups of caffeate and active site polar residues; c) the moiety oxychromenylium from anthocyanins which is relevant to the docking stabilization, and gives to this kind of compounds enough interaction capacity expressed in their higher scores. Also, important interactions with residues that are relevant to the catalytic function of the XO were observed.

The combination of in vitro and in silico techniques and procedures applied here to study the nature of enzyme inhibition mechanisms and biological activities helped us to comprehend the antioxidant functionality that a mixture of ex- 
tracts obtained from two natural products may have. We believe that the synergy between both kinds of methodologies is fundamental for the discovering of new drugs, and in this case, of potential additives for functional foods. In effect, grape pomace could constitute a cheap source of dietary antioxidants because they are secondary products of wine manufacturing. In the case of propolis, this product is consumed in small quantities by the population, so these results could constitute a good opportunity to add value to it.

In summary, according to our results we postulate that, either by inhibiting $\mathrm{XO}$ or by the scavenging of free radicals, propolis and grape pomace extracts may reduce the amount of free radicals and uric acid deposits. Thus, these natural products may be considered as functional foods and good candidates to develop phytopharmaceuticals for gout disease treatment.

\section{Acknowledgements}

This work was supported by Proyecto de Desarrollo de Ciencias Básicas (PEDECIBA) and Comisión Sectorial de Investigación Científica (CSIC), Universidad de la República-Uruguay. The authors also wish to thank Profs. Karen Ovsejevi and Carmen Manta, Cátedra de Bioquímica-Facultad de Química, Universidad de la República-Uruguay and Bodegas Carrau S. A. forprovidingthe grape pomacesamples.

\section{Conflicts of Interest}

The authors declare no conflicts of interest regarding the publication of this paper.

\section{References}

[1] Lin, S., Zhang, G., Liao, Y. and Pan, J. (2015) Inhibition of Chrysin on Xanthine Oxidase Activity and Its Inhibition Mechanism.International Journal of Biological Macromolecules, 81, 274-282. https://doi.org/10.1016/j.ijbiomac.2015.08.017

[2] Ojha, R., Singh, J., Ojha, A., Singh, H., Sharma, S. and Nepali, K. (2017) An Updated Patent Review: Xanthine Oxidase Inhibitors for the Treatment of Hyperuricemia and Gout (2011-2015). Expert Opinion on Therapeutic Patents, 27, 311-345. https://doi.org/10.1080/13543776.2017.1261111

[3] Maritim, A.C., Sanders, R.A. and Watkins, J.B. (2003) Diabetes, Oxidative Stress, and Antioxidants: A Review. Journal of Biochemical and Molecular Toxicology, 17, 24-38. https://doi.org/10.1002/jbt.10058

[4] Uttara, B., Singh, A.V., Zamboni, P. and Mahajan, R.T. (2009) Oxidative Stress and Neurodegenerative Diseases: A Review of Upstream and Downstream Antioxidant Therapeutic Options. Current Neuropharmacology, 7, 65-74. https://doi.org/10.2174/157015909787602823

[5] Adly, A.A.M. (2010) Oxidative Stress and Disease: An Updated Review. Research Journal of Immunology, 3, 129-145. https://doi.org/10.3923/rji.2010.129.145

[6] Liochev, S.I. (2013) Reactive Oxygen Species and the Free Radical Theory of Aging. Free Radical Biology and Medicine, 60, 1-4. https://doi.org/10.1016/j.freeradbiomed.2013.02.011 
[7] Qian, X., Nie, X., Yao, W., Klinghammer, K., Sudhoff, H., Kaufmann, A.M. and Albers, A.E. (2018) Reactive Oxygen Species in Cancer Stem Cells of Head and Neck Squamous Cancer. Seminars in Cancer Biology, 53, 248-257. https://doi.org/10.1016/j.semcancer.2018.06.001

[8] Dong, Y., Huang, H., Zhao, M., Sun-Waterhouse, D., Lin, L. and Xiao, C. (2016) Mechanisms Underlying the Xanthine Oxidase Inhibitory Effects of Dietary Flavonoids Galangin and Pinobanksin. Journal of Functional Foods, 24, 26-36. https://doi.org/10.1016/j.jff.2016.03.021

[9] Hille, R. and Nishino, T. (1995) Flavoprotein Structure and Mechanism. Xanthine Oxidase and Xanthine Dehydrogenase. FASEB Journal, 9, 995-1003. https://doi.org/10.1096/fasebj.9.11.7649415

[10] Umamaheswari, M., Madeswaran, A. and Asokkumar, K. (2013) Virtual Screening Analysis and In-Vitro Xanthine Oxidase Inhibitory Activity of Some Commercially Available Flavonoids. Iranian Journal of Pharmaceutical Research, 12, 317-323.

[11] Wang, Y., Zhang, G., Pan, J. and Gong, D. (2015) Novel Insights into the Inhibitory Mechanism of Kaempferol on Xanthine Oxidase. Journal of Agricultural and Food Chemistry, 63, 526-534. https://doi.org/10.1021/jf505584m

[12] Santi, M.D., PaulinoZunini, M., Vera, B., Bouzidi, C., Dumontet, V., Abin-Carriquiry, A. and Ortega, M.G. (2018) Xanthine Oxidase Inhibitory Activity of Natural and Hemisynthetic Flavonoids from Gardenia oudiepe (Rubiaceae) in Vitro and Molecular Docking Studies. European Journal of Medicinal Chemistry, 143, 577-582. https://doi.org/10.1016/j.ejmech.2017.11.071

[13] Zhang, C., Wang, R., Zhang, G. and Gong, D. (2018) Mechanistic Insights into the Inhibition of Quercetin on Xanthine Oxidase. International Journal of Biological Macromolecules, 112, 405-412. https://doi.org/10.1016/j.ijbiomac.2018.01.190

[14] Cao H., Pauff J.M. and Hille R. (2014) X-Ray Crystal Structure of a Xanthine Oxidase Complex with the Flavonoid Inhibitor Quercetin. Journal of Natural Products, 77, 1693-1699. https://doi.org/10.1021/np500320g

[15] Silva, V., Genta, G., Moler, M., Masner, M., Thomson, L., Romero, R.R., Fernandes, D., Laurindo, F., Heinzen, H., Fierro, W. and Denicola, A. (2011) Antioxidant Activity of Uruguayan Propolis. In Vitro and Cellular Assays. Journal of Agricultural and Food Chemistry, 59, 6430-6437. https://doi.org/10.1021/jf201032y

[16] Yoshizumi, K., Nishioka, N. and Tsuji, T. (2005) Xanthine Oxidase Inhibitory Activity and Hypouricemia Effect of Propolis in Rats. Journal of the Pharmaceutical Society of Japan, 125, 315-321. https://doi.org/10.1248/yakushi.125.315

[17] Yu, J. andAhmedna, M. (2013) Functional Components of Grape Pomace: Their Composition, Biological Properties and Potential Applications. International Journal of Food Science and Technology, 48, 221-237. https://doi.org/10.1111/j.1365-2621.2012.03197.x

[18] Dumitriu, D., Peinado, R.A., Peinado, J. and De Lerma, N.L. (2015) Grape Pomace Extract Improves the in Vitro and in Vivo Antioxidant Properties of Wines from Sun Light Dried Pedro Ximénez Grapes. Journal of Functional Foods, 17, 380-387. https://doi.org/10.1016/j.jff.2015.06.003

[19] Amico, V., Napoli, E.M., Renda, A., Ruberto, G., Spatafora, C. and Tringali, C. (2004) Constituents of Grape Pomace from the Sicilian Cultivar "NerelloMascalese”. Food Chemistry, 88, 599-607. https://doi.org/10.1016/j.foodchem.2004.02.022

[20] Boido, E., Alcalde-Eon, C., Carrau, F., Dellacassa, E. and Rivas-Gonzalo, J.C. (2006) Aging Effect on the Pigment Composition and Color of Vitis vinifera L. Cv. Tannat 
Wines. Contribution of the Main Pigment Families to Wine Color. Journal of Agricultural and Food Chemistry, 54, 6692-6704. https://doi.org/10.1021/jf061240m

[21] Silva, V., Genta, G., Möller, M.N., Masner, M., Thomson, L., Romero, N. and Dajas, F. (2006) Changes in Antioxidant Capacity of Tannat Red Wines during Early Maturation. Journal of Agricultural and Food Chemistry, 59, 6430-6437. https://doi.org/10.1021/jf201032y

[22] Echeverry, C., Arredondo, F., Martínez, M., Abin-Carriquiry, J.A., Midiwo, J. and Dajas, F. (2014) Antioxidant Activity, Cellular Bioavailability, and Iron and Calcium Management of Neuroprotective and Nonneuroprotective Flavones. Neurotoxicity Research, 27, 31-42. https://doi.org/10.1007/s12640-014-9483-y

[23] Alvareda, E., Miranda, P., Espinosa, V., Pardo, H., Aguilera, S. and Paulino Zunini, M. (2015) Antiinflamatory Activity of Phenolic Compounds Extracted from Uruguayan Propolis and Grape. Journal of Biomolecular Structure and Dynamics 33, 129. https://doi.org/10.1080/07391102.2015.1032833

[24] Paulino, M., Alvareda, E., Iribarne, F., Miranda, P., Espinosa, V., Aguilera, S. and Pardo, H. (2016) Toward the Understanding of the Molecular Basis for the Inhibition of COX-1 and COX-2 by Phenolic Compounds Present in Uruguayan Propolis and Grape Pomace. Journal of Biomolecular Structure and Dynamics, 34, 2643-2657. https://doi.org/10.1080/07391102.2015.1124808

[25] Singleton, V.L., Orthofer, R. and Lamuela-Raventos, R.M. (1999) Analysis of Total Phenols and Other Oxidation Substrates and Antioxidants by Means of Folin-Ciocalteau Reagent. Methods in Enzimology, 299, 153-178. https://doi.org/10.1016/S0076-6879(99)99017-1

[26] Shimada, K., Fujikawa, K., Yahara, K. and Nakamura, T. (1992) Antioxidative Properties of Xanthan on the Autoxidation of Soybean Oil in Cyclodextrin Emulsion. Journal of Agricultural and Food Chemistry, 40, 945-948. https://doi.org/10.1021/jf00018a005

[27] Molecular Operating Environment (MOE ${ }^{\mathrm{TM}}$ ) (2015) Chemical Computing Group Inc., Montreal.

[28] Farre, R., Frasquet, I. and Sanchez, A. (2004) El propolis y la salud. Ars Pharmaceutica, 45, 21-43.

[29] Paulino-Zunini, M., Rojas, C., Depaula, S., Elingold, I., Alvareda, E., Casanova, M.B., Iribarne, F. and Dubin, M. (2010) Phenolic Contents and Antioxidant Activity in Central Southern Uruguayan Propolis Extracts. Journal of the Chilean Chemical Society, 551, 141-146. https://doi.org/10.4067/S0717-97072010000100033

[30] Alencar, S.M., Oldoni, T.L., Castro, M.L., Cabral, I.S., Costa-Neto, C.M., Cury, J.A., Rosalen, P.L. and Ikegaki, M. (2007) Chemical Composition and Biological Activity of a New Type of Brazilian Propolis: Red Propolis. Journal of Ethnopharmacology, 113, 278-283. https://doi.org/10.1016/j.jep.2007.06.005

[31] Falcão, S.I., Vilas-Boas, M., Estevinho, L.M., Barros, C., Domingues, M.R. and Cardoso, S.M. (2010) Phenolic Characterization of Northeast Portuguese Propolis: Usual and Unusual Compounds. Analytical and Bioanalytical Chemistry, 396, 887-897. https://doi.org/10.1007/s00216-009-3232-8

[32] Halgren, T.A. (1999) MMFF VI. MMFF94s Option for Energy Minimization Studies. Journal of Computational Chemistry, 20, 720-729. https://doi.org/10.1002/(SICI)1096-987X(199905)20:7<720::AID-JCC7>3.0.CO;2-X

[33] Hille, R. (2006) Structure and Function of Xanthine Oxidoreductase. European Journal of Inorganic Chemistry, 2006, 1913-1926. https://doi.org/10.1002/ejic.200600087 
[34] Vera, B., Vázquez, K., Mascayano, C., Tapia, R.A., Espinosa, V., Soto-Delgado, J., Salas, C.O. and Paulino, M. (2016) Structural Analysis and Molecular Docking of Trypanocidal Aryloxy-Quinones in Trypanothione and Glutathione Reductases: A Comparison with Biochemical Data. Journal of Biomolecular Structure and Dynamics, 35, 1785-1803. https://doi.org/10.1080/07391102.2016.1195283

[35] Haidari, F., Mohammad Shahi, M., Keshavarz, S.A. and Rashidi, M.R. (2009) Inhibitory Effects of Tart Cherry (Prunuscerasus) Juice on Xanthine Oxidoreductase Activity and Its Hypouricemic and Antioxidant Effects on Rats. Malaysian Journal of Nutrition, 15, 53-64.

[36] Bräunlich, M., Slimestad, R., Wangensteen, H., Brede, C., Malterud, K.E. and Barsett, H. (2013) Extracts, Anthocyanins and Procyanidins from Aronia melanocarpa as Radical Scavengers and Enzyme Inhibitors. Nutrients, 5, 663-678.

https://doi.org/10.3390/nu5030663

[37] Zhang, Z.C., Wang, H., Zhou, Q., Hu, B., Wen, J.H. and Zhang, J.L. (2017) Screening of Effective Xanthine Oxidase Inhibitors in Dietary Anthocyanins from Purple Sweet Potato (Ipomoea batatas L. Cultivar Eshu No. 8) and Deciphering of the Underlying Mechanisms in Vitro. Journal of Functional Foods, 36, 102-111. https://doi.org/10.1016/j.jff.2017.06.048

[38] Huang, S., Zhang, C.-P., Wang, K., Li, G. and Hu, F.-L. (2014) Recent Advances in the Chemical Composition of Propolis. Molecules, 19, 19610-19632.

https://doi.org/10.3390/molecules191219610

[39] Echeverry, C., Ferreira, M., Reyes-Parada, M., Abin-Carriquiry, J.A., Blasina, F., González-Neves, G. and Dajas, F. (2005) Changes in Antioxidant Capacity of Tannat Red Wines during Early Maturation. Journal of Food Engineering, 69, 147-154. https://doi.org/10.1016/j.jfoodeng.2004.08.005

[40] Shin, J.-H., Chun, K.S., Na, Y.-G., Song, K.-H., Kim, S., Lim, J.-S. and Kim, G.-H. (2015) Oxidative Medicine and Cellular Longevity, 15, Article ID: 906787.

[41] Wróblewski, K., Muhandiram, R., Chakrabartty, A. and Bennick, A. (2001) The Molecular Interaction of Human Salivary Histatins with Polyphenolic Compounds. European Journal of Biochemistry, 268, 4384-4397. https://doi.org/10.1046/j.1432-1327.2001.02350.x 


\section{Supplementary Information (SI)}

Table S1. Xanthine oxidase docking scores (Affinity $\Delta \mathrm{G}$ algorithm), of the 60 best docked compounds. A PubChem code as a compound identifier (CID) is given in parentheses, ( ${ }^{*} \mathrm{CID}$ refers to a CID parent code). Origin of compounds denoted as "P" (Propolis) or "GP” (grapes). Basic structural scaffolds as follows: PHE AC: Phenolic acids; PROCY: Procyanidins; ANTHO: Anthocyanins; FLAV: Flavonoid; STYL: Stylbene; CAT: Catechin.

\begin{tabular}{|c|c|c|c|}
\hline Name** & XO Scores $\left(\mathrm{kcal} \cdot \mathrm{mol}^{-1}\right)$ & Origin ${ }^{* *}$ & Structure ${ }^{* *}$ \\
\hline (E)-isoprenylcaffeate (5281790) & -5.7884 & $\mathrm{P}$ & PHE AC Ester \\
\hline Cyanidin-3-O-(6-(E)-p-coumaroyl)-glucoside (5282067) & -5.6533 & GP & ANTHO \\
\hline Pyruvic derivative of malvidin-3-O-glucoside (443652) & -5.3929 & GP & ANTHO \\
\hline Pyruvic derivative of delphinidin-3-O-(6-p-coumaroyl)-glucoside (15922818) & -5.3318 & GP & ANTHO \\
\hline (Z)-isoprenylcaffeate (5281790) & -5.3258 & $\mathrm{P}$ & PHE AC Ester \\
\hline Malvidin-3-O-(6-(E)-p-coumaroyl)-glucoside-4-vinylcatechol $\left({ }^{*} 44256995\right)$ & -5.3088 & GP & ANTHO \\
\hline Petunidin-3-O-glucoside-4-vinylcatechol $\left({ }^{*} 443651\right)$ & -5.1905 & GP & ANTHO \\
\hline (E)-phenethylcaffeate (CAPE) (5881787) & -5.1849 & $\mathrm{P}$ & PHE AC Ester \\
\hline 5-methoxypinobanksin-3-O-pentanoate (147459) & -5.1234 & GP & FLAV Ester \\
\hline (E)-cinnamylcaffeate (5281787) & -5.0530 & $\mathrm{P}$ & PHE AC Ester \\
\hline Pinobanksin-3-O-acetate (148556) & -5.0341 & $\mathrm{P}$ & FLAV Ester \\
\hline Malvidin-3-O-(6-acetyl)-glucoside-4-vinylguaiacol $\left({ }^{*} 44257037\right)$ & -4.9974 & GP & ANTHO \\
\hline Peonidin-3-O-glucoside-4-vinylguaiacol $\left({ }^{\star} 443654\right)$ & -4.9925 & GP & ANTHO \\
\hline Petunidin-3-O-(6-(Z)-p-coumaroyl)-glucoside (44256963) & -4.9893 & GP & ANTHO \\
\hline Malvidin-3-O-glucoside-4-vinylguaiacol (44257037) & -4.9663 & GP & ANTHO \\
\hline (E)-caftaric acid (6440397) & -4.9550 & GP & PHE AC \\
\hline Delphinidin-3-O-(6-(E)-p-coumaroyl)-glucoside (15922818) & -4.9364 & GP & ANTHO \\
\hline Procyanidin B2 3’-gallate $\left({ }^{*} 122738\right)$ & -4.9230 & GP & PROCY \\
\hline (E)-resveratrol (445154) & -4.7791 & $\mathrm{P} / \mathrm{GP}$ & STYL \\
\hline Malvidin-3-O-(6-acetyl)-glucoside-4-vinylphenol $\left({ }^{\star} 44257035\right)$ & -4.7603 & GP & ANTHO \\
\hline (E)-isoprenyl-p-coumarate $\left({ }^{*} 637542\right)$ & -4.7592 & $\mathrm{P}$ & PHE AC Ester \\
\hline Delphinidin-3-O-(6-(Z)-p-coumaroyl)-glucoside (44256898) & -4.7251 & GP & ANTHO \\
\hline Malvidin-3-O-glucoside-4-vinylcatechol (44257036) & -4.7080 & GP & ANTHO \\
\hline Petunidin-3-O-glucoside-4-vinylphenol $\left({ }^{*} 443651\right)$ & -4.6519 & GP & ANTHO \\
\hline Peonidin-3-O-(6-(E)-p-coumaroyl)-glucoside (443654) & -4.6496 & GP & ANTHO \\
\hline Vitisin B of malvidin-3-O-(6-p-coumaroyl)-glucoside (71308302) & -4.6318 & GP & ANTHO \\
\hline Malvidin-3-O-(6-(E)-p-coumaroyl)-glucoside-4-vinylphenol $\left({ }^{*} 44256995\right)$ & -4.5634 & GP & ANTHO \\
\hline Quercetin-7-O-neohesperidoside $\left({ }^{\star} 5280343\right)$ & -4.5625 & GP & FLAV Glyc \\
\hline Peonidin-3-O-glucoside-4-vinylphenol $\left({ }^{*} 443654\right)$ & -4.5593 & GP & ANTHO \\
\hline (E)-bencylcaffeate (5919576) & -4.5426 & $\mathrm{P}$ & PHE AC \\
\hline Petunidin-3-O-(6-(E)-p-coumaroyl)-glucoside (176449) & -4.5289 & GP & ANTHO \\
\hline Cyanidin-3-O-(6-(Z)-p-coumaroyl)-glucoside (5282067) & -4.4787 & GP & ANTHO \\
\hline (Z)-isoprenyl-p-coumarate $\left({ }^{\star} 637542\right)$ & -4.4233 & GP & PHE AC Ester \\
\hline
\end{tabular}




\section{Continued}

Pyruvic derivative of delphinidin-3-O-glucoside (165558)

$-4.3155$

$-4.3068$

Pyruvic derivative of peonidin-3-O-(6-acetyl)-glucoside

Pyruvic derivative of petunidin-3-O-(6-p-coumaroyl)-glucoside (72193651)

(Z)-caftaric acid (72551521)

Delphinidin-3-O-(6-acetyl)-glucoside (15385440)

Quercetin-3-O-galactoside (5281643)

Pyruvic derivative of peonidin-3-O-glucoside (443654)

3.4-dihydroxyvinylbencene (151398)

Malvidin-3-O-glucoside-4-vinylphenol (44257035)

(E)-caffeic acid (717531)

Pyruvic derivative of malvidin-3-O-(6-p-coumaroyl)-glucoside (72193651)

Vitisin B of malvidin-3-O-(6-acetyl)-glucoside $\left({ }^{\star} 71308302\right)$

Apigenin (5280443)

Methylgallate (7428)

Procyanidin B3 (4R-8 (+)C(-)C) (146798)

Delphinidin-3-O-glucoside-4-vinylphenol $\left({ }^{\star} 443650\right)$

Pyruvic derivative of delphinidin-3-O-(6-acetyl)-glucoside (15385440)

(+)-gallocatechin (65084)

Isorhamnetin-3-O-glucoside (5318645)

Procyanidin B8 (4R-6 (+)C(+)E) (130556)

Fisetin (5281614)

Malvidin-3-O-(6-acetyl)-glucoside-4-vinylcatechol ( $\left.{ }^{\star} 44257036\right)$

Peonidin-3-O-glucoside (443654)

Oroxylin A (5320315)

Petunidin-3-O-(6-acetyl)-glucoside (44256961)

Naringin (442428)

Malvidin-3-O-(6-(E)-caffeoyl)-glucoside (44256989)
$-4.2970$

$-4.2795$

$-4.2767$

$-4.2540$

$-4.2522$

$-4.2318$

$-4.2188$

$-4.2118$

$-4.2087$

$-4.2065$

$-4.1989$

$-4.1983$

$-4.1970$

$-4.1664$

$-4.1439$

$-4.1433$

$-4.1284$

$-4.1111$

$-4.1051$

$-4.0565$

$-4.0496$

$-4.0489$

$-4.0434$

$-4.0359$

$-4.0332$
GP

GP

GP

GP

GP

GP

GP

P

GP

P

GP

GP

P

GP

GP

GP

GP

GP

GP

GP

P

GP

GP

GP

GP

P

GP
ANTHO

ANTHO

ANTHO

PHE AC

ANTHO

FLAV Glyc

ANTHO

STYL

ANTHO

PHE AC

ANTHO

ANTHO

FLAV

FLAV

PROCY

ANTHO

ANTHO

CAT

FLAV Glyc

PROCY

FLAV

ANTHO

ANTHO

ANTHO

ANTHO

FLAV

ANTHO

${ }^{*}$ Names, origin and structures were taken from Paulino et al. [24] with permission of all co-authors. 